San Jose State University

SJSU ScholarWorks

Master's Projects

Master's Theses and Graduate Research

2005

\title{
Evaluation of the Effectiveness of a Diabetes Managment Training Program for Unlicensed Assistive Personnel in Schools
}

\author{
Elaine M. Gomer \\ San Jose State University
}

Follow this and additional works at: https://scholarworks.sjsu.edu/etd_projects

Part of the Pediatric Nursing Commons

\section{Recommended Citation}

Gomer, Elaine M., "Evaluation of the Effectiveness of a Diabetes Managment Training Program for Unlicensed Assistive Personnel in Schools" (2005). Master's Projects. 764.

DOI: https://doi.org/10.31979/etd.adbu-2swk

https://scholarworks.sjsu.edu/etd_projects/764

This Master's Project is brought to you for free and open access by the Master's Theses and Graduate Research at SJSU ScholarWorks. It has been accepted for inclusion in Master's Projects by an authorized administrator of SJSU ScholarWorks. For more information, please contact scholarworks@sjsu.edu. 


\section{SAN JOSE STATE UNIVERSITY SCHOOL OF NURSING}

\section{MAASTER'S PROGRAM PROJECT OPTION (PLAN B) PROJECT SIGNATURE FORM}

STUDENT NAME

Elaine Gomer

SEMESTER ENROLLED Fall 2005

TITLE OF PROJECT Evaluation of the

Effectiveness of A Diabetes

Management Training Program for
Unlicensed Assistive Personnel in Schools
NAmE OF JOURNAL The Journal of School Nursing

The project and manuscript have been successfully completed and meet the standards of the School of Nursing at San Jose State University. The project demonstrates the application of professional knowledge, clinical expertise, and scholarly thinking. An abstract of the project and two copies of the manuscript are attached.

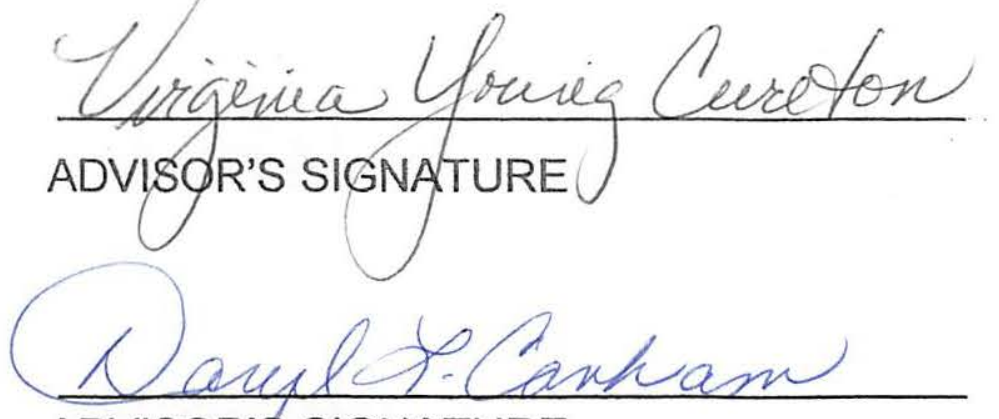

ADVISOR'S SIGNATURE



DATE

Please submit this form to the Graduate Coordinator. Attach abstract, two copies of the manuscript, and documentation of submission to the journal (i.e., postal receipt). 
EVALUATION OF THE EFFECTIVENESS OF A DIABETES

MANAGEMENT TRAINING PROGRAM FOR UNLICENSED

\title{
ASSISTIVE PERSONNEL IN
}

\section{SCHOOLS}

\section{Authors}

\author{
Elaine M.Gomer, RN, BSN
}

School Nurse, Morgan Hill Unified School District

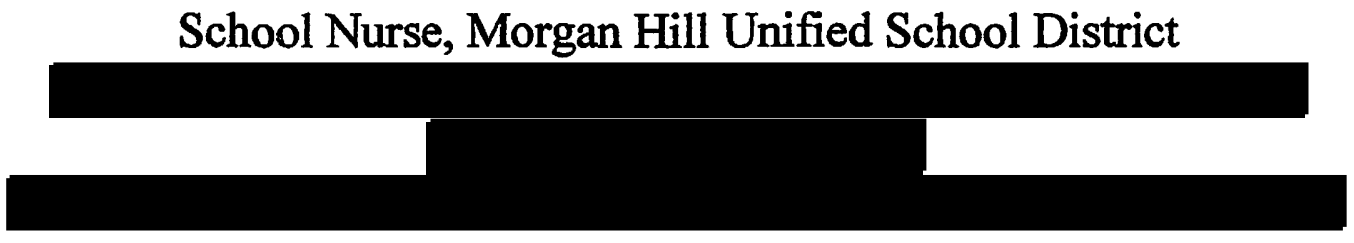

Professor Virginia Young Cureton, RN, DPH

School of Nursing, San Jose State University

One Washington Square, San Jose, CA. 95192-0057

(408) 924-3163 FAX (408) 268-2545

Associate Professor Daryl L. Canham, EdD, RN, BC

Coordinator, SNCP

School of Nursing, San Jose State University

One Washington Square, San Jose, CA. $95192-0057$ (408) 924-1323

canham@son.sjsu.edu

December 2, 2005 7:45 pm 
ABSTRACT: This study evaluated the diabetes management-training program for unlicensed assistive personnel (UAP). The purpose of the program was to prepare UAP to recognize and respond to the health care needs of students diagnosed with diabetes mellitus. Twenty UAP participated. Teaching strategies were based on Albert Bandura's social learning theory: Two instruments were administered before and after the program. One was a 10-question survey addressing self-efficacy; the other was a 10 question multiple-choice test measuring knowledge of diabetes. Results of paired $t$ tests indicate consistent and significant improvement in both perceptions of self-efficacy and knowledge $(p<.05)$. This program provides a framework for school nurses training UAP that assist in the health care of students with diabetes. It is important that school nurses capitalize on the contribution that informed confident UAP might contribute to the provision of safe quality care to students with diabetes.

KEY WORDS: diabetes, school nursing, self-efficacy, social learning theory, training program, unlicensed assistive personnel (UAP). 


\section{INTRODUCTION}

School nurses have numerous and diverse responsibilities to provide support services to children with diabetes and to the staff members caring for them. The second most prevalent rate of chronic disease behind asthma, diabetes is a health condition all school districts must be prepared to deal with (American Diabetes Association, 2003). To provide the appropriate care of the student with diabetes it is crucial that school personnel: (a) have sufficient knowledge about diabetes; and (b) are trained in diabetes management thus allowing students full participation in school, minimizing interruption of school activities, and assuring a safe environment. While all school staff members cannot be trained in diabetes management, there should be a minimum vital number of personnel who are knowledgeable. Key staff members, mostly unlicensed assistive personnel (UAP) who regularly interact with the student must receive appropriate training on the student's needs, health precautions, and emergency care. The purpose of this study was to measure the effect of a diabetes management-training program (DMTP) for UAP in the school setting on knowledge and self-efficacy. Implementation of a DMTP for school personnel benefits students with diabetes by improving attendance and academic performance, maximizing participation in school activities, and reducing the progression of chronic complications.

\section{Research Question}

What is the effect of a DMTP based on the social learning theory of Albert Bandura and designed for UAP on knowledge and self-efficacy in responding to the health needs of diabetic students? 
Literature Review

Diabetes is the sixth leading cause of death in the United States with an incidence rate of $6.3 \%$ of the population (Centers For Disease Control and Prevention, [CDC], 2002). Nationally, approximately 1 in every 400-500 children and adolescents has Type I diabetes and approximately 13,000 school- age children are diagnosed every year (National Diabetes Educational Program, 2003). The incidence of diabetes is the highest incidence among all childhood chronic diseases.

Type I diabetes management is complicated and demanding, requiring daily regulation and self-monitoring. Type I diabetes can be associated with one or several serious complications. Chronic micro-vascular complications can affect every body system. The cumulative effect of poor diabetes management is damaging. Heart disease and stroke account for $65 \%$ of deaths among diabetics (CDC, 2002).

Another complication of diabetes is the increased susceptibility to other illnesses and the greater than average time required to recover from illness. Nichols and Norris (2002) cited a study by Vetiska and colleagues that found students with diabetes missed an average of 6 more school days each year then their siblings who did not have diabetes. The psychological stress and anxiety related to falling behind in school work, the negative effects on self-esteem by appearing different, and the interference with peer relations caused by diabetes management are barriers to optimal academic achievement. Kathleen Thies (1999) reported that $45 \%$ of students with chronic illness fell behind in schoolwork and that falling behind in schoolwork contributed to an aversion to school. Diabetes has been shown to contribute to both impaired planning of tasks and attention to 
detail, slower response time, fatigue, confusion, impaired spatial abilities, eye damage, and difficulties with visual scanning, and reading.

The population of children with diabetes in schools continues to grow and school personnel report feeling inadequate about their knowledge and ability to respond to the health needs of diabetic students or to diabetic emergencies (Lewis, Powers, Goodenough, \& Poth, 2003). Schools must accommodate children's medical conditions as mandated by Public Law, Section 504 of the Rehabilitation Act of 1973, the Individuals with Disabilities and Education Act of 1991; and The Americans With Disabilities Act of 1992 (Juvenile Diabetes Research Foundation, 2005).

Accommodation denotes more than allowing students to test blood glucose and to take insulin when needed. The term also relates to school districts' responsibility to provide for the safety of students with diabetes and ensure that they are ready to learn and to fully participate in school activities. Despite the federal mandate, $3 \%$ of schools studied reported that school children with diabetes were not allowed to test blood glucose while at school and that blood glucose supplies were not provided (Lewis et al.).

Approximately $20 \%$ of parents of children with diabetes reported that their child was not allowed to participate in certain school activities without specific restrictions. These restrictions are discriminatory and are based on a lack of knowledge of this chronic condition. Schools are financially unable to employ adequate numbers of school nurses to provide care for and to be regularly available to students. Therefore, students must rely on unlicensed school personnel to help meet their health needs. In a study investigating the adequacy of in-school support for diabetic children, researchers found that $10 \%$ of schools did not have a policy for management of school children with 
diabetes (Lewis et al.). In another study, $30 \%$ of parents of children with diabetes responded that the in-school support for their children was inadequate. In addition, $13 \%$ of parents reported a lack of school personnel trained in diabetes management (HayesBohn, Neumark-Sztainer, Mellin, \& Patterson, 2004).

The American Diabetes Association (2001) recommends that schools provide diabetes education for all school personnel. Improvement in parent satisfaction has been reported when school personnel are informed and trained in diabetes management (Lewis et al., 2003). Nichols and Norris (2002) reviewed four studies to evaluate the effectiveness of diabetes education of school personnel. The authors concluded that teacher knowledge was inadequate and that students' health and safety were at risk.

Siminerio and Koerbel (2000) investigated the effectiveness of diabetes education programs for school personnel and found that these types of programs are highly effective in increasing efficacy levels. The results demonstrated statistically significant improved scores on general knowledge following the program. However, study participants voiced a serious concern regarding the lack of information and training provided to ancillary school personnel.

As advocates for students with health conditions school nurses must promote the use of student health care plans, remind school district personnel of their responsibilities to students with health conditions, and coordinate diabetes management, education, and training. Researchers have documented the necessity for school nurses to designate diabetes management tasks to UAP following adequate training and validation of competence. In a position statement published by the American Academy of Pediatrics 
(2001), the school nurse should supervise the education of 2 or more staff members per school to handle emergencies.

VanCura and Gunchick (1997) described five key components for effectively working with UAP. These components are: (a) management support, (b) role competency, (c) appropriate delegation, (d) teamwork, and (e) effective communication. A DMTP should include the information required to: understand diabetes, manage diabetes care in schools, including hyperglycemia and hypoglycemia; and to administer glucagon when indicated. Following a survey of pediatric diabetic educators, Jameson (2004) found that following implementation of diabetes training programs in schools, knowledge retention was excellent, improved care for students was noted, and trainees displayed good participation and provided positive feedback.

School nurses are assuming more responsibility for supervising the care that UAP provide. When training UAP to provide specific nursing activities and tasks, the school nurse is responsible and accountable for the care provided. State law or nurse practice regulations allowing delegation of medication administration to UAP may not provide clear guidelines for administration of emergency medications, such as giving an injection of glucagon.

Reutzel and Patel (2001) conducted exploratory research to gain an understanding of the medication management problems experienced by school nurses. They collected information electronically from subscribers to a school nurse listserv. There was not a consensus among participants on whether it is appropriate to train or allow UAP to administer glucagon in an emergency situation. The school nurses expressed the need for further information regarding laws and regulations, guidelines, policies, and practice. 
The conflict between school nurse professional regulations of practice and state education codes is a controversial issue that is managed differently from state to state as well as by school districts within each state.

A review of the literature clearly demonstrates the need to properly train UAP to safely care for students and emphasizes the need to develop guidelines and standards for training programs. Cynthia Corbett (1999) found levels of self-efficacy among students with diabetics were higher following a diabetes management program with efficacyenhancing interventions. Overall, the participants' confidence level to self-manage their diabetes increased significantly following the intervention.

Litarowsky, Murphy, and Canham (2004) conducted a study to evaluate the effectiveness of an anaphylaxis-training program for UAP using strategies designed to enhance self-efficacy. Findings support the hypothesis that a training program based on social learning theory can increase the knowledge and self-efficacy of UAP for responding to an anaphylactic emergency.

Theoretical Framework

Albert Bandura (1994) developed a theory of self-efficacy that provides an effective conceptual framework to guide development of a DMTP designed for UAP. Self-efficacy theory is based on an individual's belief system, which states that a person's understanding of how the world works and what his/her place is in the world affects how he/she responds to the world. Self-efficacy beliefs influence the choices individuals make and the actions they take.

Individuals develop perceptions as a result of past successes and failures. Confident individuals expect successful results; conversely those individuals who lack 
confidence have lower levels of self-efficacy and will expect poor results. Self-efficacy beliefs also influence the degree of endeavor people put forth to accomplish a task and how long they persist and persevere at a task when encountering deterrents. The stronger the perceived self-efficacy, the higher are the goal standards and the more energetic the commitment to these goals.

Bandura (1994) writes that feelings of competency come from four major sources: first, mastery experiences; second, vicarious experiences; third, verbal persuasion; and fourth, physiological states. Mastery is the most effective way to build a strong sense of self-efficacy and failures tend to decrease levels of self-efficacy. However, if people experience only success, they may become easily thwarted by failure and they may never have the opportunity to develop a sense of resiliency.

The second source of self-efficacy results from vicarious experiences that are derived from social models. Seeing someone similar to oneself succeed leads to the belief that one is capable of success. The third source of self-efficacy occurs when verbal persuasion originates from encouraging remarks one hears that he/she is capable of mastering tasks. These verbal remarks motivate individuals to put forth greater effort to accomplish tasks. The fourth source of self-efficacy originates from physiological states that refer to an individual's perception of his/her physical and emotional state in judging one's own capabilities. Positive perceptions of emotions will enhance feelings of selfefficacy and dejected emotions will decrease feelings of self-efficacy.

In training UAP, the school nurse is challenged with issues of liability, fear, and anxiety related to UAP misjudging a situation or the misinterpretation of symptoms that could result in the delivery of the wrong action and possibly causing harm to a student. 
UAP frequently voice distress and feelings of inadequacy concerning their ability to manage procedures under pressing situations. To successfully train UAP, the school nurse must present a program that generates interest in the topic issue and that motivates UAP to believe that they are capable of responding appropriately. Application of the Theoretical Framework in this Study

Prior to the DMTP presentation, the nurse educator will administer a pre-test and questionnaire to discover UAP baseline knowledge and perceived self-efficacy in dealing with diabetes. This stage of the intervention will assist the UAP in determining the significance of the problem to themselves and in evaluating their own expectations about themselves. This educational program on diabetes training for UAP based on Bandura's (1994) theory will utilize role models to provide vicarious experiences that create and strengthen self-beliefs of self-efficacy. By watching the school nurse, the trainee will observe the behavior that will achieve the desired result. This statement is especially true when the school nurse demonstrates proper injection of glucagon and when the school nurse supervises UAP blood glucose monitoring.

Bandura (2001) writes about the importance of peers in broadening self-efficacy. They serve as a major influence in the development and validation of self-efficacy. Part of the training program includes hands on practice with equipment used to control and treat diabetes. If UAP see their peers mastering the tasks, the UAP will more strongly belief that they possess the capabilities to master similar activities.

Verbal encouragement can be an effective technique to increase self-efficacy by persuading people that they possess the abilities and are capable of mastering given 
activities. As The health expert in the school, the school nurse's positive feedback will be eminently persuasive.

As mentioned earlier, the fourth source of self-efficacy is physiological arousal. Diabetes can lead to life threatening situations for the students and many UAP are concerned with liability. Physiological states such as anxiety and stress have a potent influence on behavior, the motivation to act, and on perception of self-efficacy. Part of the training program provides time to discuss the stress and anxiety UAP typically feel when dealing with emergent situations. In stressing the effectiveness of treatment and emphasizing the unlikely occurrence of harming a student with treatment, the school nurse will mitigate the UAP anxiety levels.

This educational program was designed to follow Bandura's conceptual format by: (a) using role models, (b) working with peers, (c) giving demonstrations and return demonstrations, (d) using verbal persuasion, and (e) providing hands-on practice. Bandura's concepts provide insight on how school nurses as health educators can positively influence behavior change by supporting and encouraging a strong sense of self-efficacy in UAP.

\section{METHODOLOGY}

Hypothesis

Implementation of a diabetes management-training program for UAP based on Albert Bandura's social learning theory with the purpose of improving the UAP's response to the health needs of students with diabetes, will increase UAP perception of self-efficacy and will improve test scores on knowledge of diabetes. 
Research Design and Sample

A pre-experimental design using a diabetes knowledge test and a self-efficacy survey administered prior to the training program and repeated at the conclusion of the presentation was employed in this study. The power point presentation was adopted from Pediatric Education for Diabetes in schools (Zombeck, 2001). A brief statement describing the study was given at the beginning of the presentation and only those UAP who signed a formal consent form participated in the research study. Permission to present and evaluate the DMTP was obtained from the Superintendent of the School District and from the San Jose State University, Institutional Review Board prior to implementation.

Notices were distributed to all schools early in the school year describing the program topic, dates, and times. Health clerks, school secretaries, student supervisors, teachers, and administrators were invited to attend.

Demographic data that was requested included: employment status, age group, student population currently working with, and total number of years working for a school district. School personnel represented two high schools, two middle schools, and nine elementary schools. A convenience sample of 15 health clerks and 5 school secretaries, all female, completed the program. Age of participants ranged from 21 years to over 65 years of age. Participant years of experience working in a school district included: $40 \%$ of participants had less than 5 years experience, $40 \%$ of participants had 5 to 9 years, $15 \%$ of participants had 10 to 14 years experience, and $5 \%$ of participants had 15 to 20 years of experience. 
The school nurse conducted the presentation of DMTP for UAP at the school district office boardroom. A cover page on the tests and surveys were labeled with the last 4 digits of each participant's social security number. Pre DMTP tests and surveys were matched with post DMTP tests and surveys, they were attached, and the cover pages with the identifying numbers were removed and destroyed. There was no way to determine the identity of the UAP who took each test.

Intervention: Diabetes Management-Training Program

The school nurse presented the 2.5 hour diabetes-training program for school personnel utilizing a PowerPoint format. Topics included: (a) definitions, causes, and symptoms of hypoglycemia and hyperglycemia, (b) prevalence, (c) types of diabetes, (d) diabetes management, (e) school accommodations, (f) blood glucose monitoring, (g) treatment of hypoglycemia and hyperglycemia, (h) insulin delivery systems, (i) role of the school nurse, and (j) the role of the UAP.

Following the presentation and discussion of the DMTP, the school nurse demonstrated diabetes management techniques. The participants were then asked to conduct hands-on practice of these techniques using simulated and real models. The participants practiced in close proximity to one another to facilitate sharing of ideas, insights, and feedback. The school nurse was present to monitor the participants' practice techniques and to answer any questions. Instrumentation

A quantitative test was used to measure knowledge level of diabetes and a survey was used to measure self-efficacy. The 10-question multiple-choice test used to measure mastery of knowledge content was created directly from the information presented in the 
program. Two other school nurses reviewed the test for accuracy and validation of the tool.

Perceived self-efficacy was measured by using a 10-item self-efficacy Likert-style survey modified from one designed by Litarowsky, Murphy, and Canham (2004) in their research study of Evaluation of an Anaphylaxis Training Program for Unlicensed Assistive Personnel. The terms "diabetes management" and "diabetic emergencies" were substituted for "allergic reactions" and "anaphylaxis" respectively. Jean Litarowsky granted written permission for modification of her self-efficacy tool for this research study. Participants were asked to rate their confidence level from 1-4 $(1=$ very confident, 2 = somewhat confident, $3=$ somewhat lacking confidence, $4=$ completely lacking confidence) regarding ability to recognize and to respond appropriately to a diabetic emergency. This pre-DMTP knowledge test and self-efficacy survey provided the pre-program data. At the conclusion of the DMTP, the diabetes knowledge test and self-efficacy survey were re-administered to participants. This post- test and post-survey provided the post-DMTP data.

Data Collection and Analysis

Two separate training sessions were conducted 1 week apart. Participants attended only one session. A morning session and an evening session were offered to facilitate work schedules. Participants represented all school sites within the school district with the exception of the continuation high school.

\section{FINDINGS}

Table 1 shows a comparison of the pre and posttest results for knowledge and self-efficacy. From the pre and post knowledge test data and from the pre and post self- 
efficacy survey the paired samples $t$ test was the statistical measure used in this study to analyze data. The maximum knowledge score possible on the pre/posttest was +10 . The mean pretest knowledge score of $7.15(\mathrm{SD}=1.8)$ increased to $8.0500(\mathrm{SD}=1.19)$ after the training intervention. Pretest scores ranged from 3 to 9 correct answers and posttest scores increased to a range from 6 to 10 correct. The highest pretest score of 9 correct answers was achieved by $20 \%$ of the subjects $(n=4)$. The greatest frequency of correct answers was $40 \%(n=8)$ of participants scoring 8 correct. The minimum score of 3 correct resulted from $10 \%(n=2)$ of participants. Following the training program the highest posttest score was 10 correct by $5 \%(n=1)$ of participants, and the highest frequency of correct answers occurred with 8 correct achieved by $40 \%(n=8)$. The minimum score increased to 6 correct answers obtained by $15 \%(n=3)$ participants. In comparing the pre-knowledge and post-knowledge test scores the mean was greater for the post scores indicating a significant increase in knowledge $(p<.05)$.

Perceived self-efficacy scores showed significant improvement following the DMTP when compared to pre-DMTP results. The statistical results demonstrated that when comparing the pre self-efficacy and the post self-efficacy scores, the mean was less (meaning greater confidence) for the post self-efficacy survey. The strongest perception of perceived self-efficacy score $=10$. The pre-training questionnaire mean score of $21.8500(\mathrm{SD}=7.64526)$ decreased to $14.20(\mathrm{SD}=3.78)$ immediately following the presentation. Pre-intervention scores ranged from 10 to 35 points whereas postintervention scores ranged from 10-23 points (indicating increased self-efficacy). Subjects scoring their perceived self-efficacy at 22 points or less included $50 \%$ of participants $(n=10)$ at pre-intervention compared to $95 \%(n=19)$ at post intervention. 
When analyzing the paired samples test, the mean for the pre knowledge/post knowledge score $=-.90000(t=-2.349)$. The mean for the pre self-efficacy/post selfefficacy score $=7.6500(t=5.691)$. Results in this study show that there is a statistically significant difference between the means for both self-efficacy and knowledge $(p<.05)$. These findings support the hypothesis that implementation of a DMTP for UAP in schools, based on the social learning theory of Albert Bandura on knowledge and selfefficacy, improved UAP perception of self-efficacy and diabetes knowledge test scores significantly.

\section{Limitations of the Study}

The limitations of this study included the inability to conduct random sampling and the lack of a control group. Findings cannot be generalized. The 2.5 hours available for conducting the program is an insufficient amount of time to properly train UAP. However, school staff members and administrators clearly objected to allowing employees more time away from schools for training purposes, citing a lack of substitute personnel to assume the participating UAP responsibilities. To hire substitutes or pay overtime to employees was viewed as financially infeasible.

Health clerks who had received some previous training in diabetes management indicated they could assimilate the information. However, secretaries and newly hired health clerks expressed feeling somewhat overwhelmed with so much information and acknowledged the need for reinforcement and follow-up. School administrators, teachers, coaches, and student supervisors failed to attend the training program though adequate notification was provided. 
IMPLICATIONS FOR SCHOOL NURSING RESEARCH AND PRACTICE

Increasing self-efficacy in conjunction with knowledge and skill provides

effective results and may be applied to other areas of training UAP. An example of how increasing knowledge and self-efficacy may improve the care and safety of other students would be for the school nurse to train UAP to assist a student who is having an asthma attack, and how to appropriately use his/her inhaler. A follow-up study to measure long term knowledge retention and maintenance of self-efficacy would help determine frequency of training and time necessary for skill practice. This study could be replicated using a random sample, or a larger sample.

Many school districts' policies and guidelines regarding accommodations for students with diabetes are vague or absent. It may only be through a Section 504 process that these accommodations are clarified. School personnel are frequently confused regarding their level of responsibility in caring for the student. School nurses can document the need for trainings, advocate for appropriate school district health and safety policies, and provide input to update and revise school district guidelines.

School nurse practice has slowly diminished over recent years as states struggle to balance the budget for public education and districts cut health services to the minimum acceptable level. Students with diabetes continue to exist in notable numbers in the school system and are entitled to federally mandated accommodations to allow equal access to education. The school nurse (if one exists) must rely on UAP to respond to the health and safety needs of the students with diabetes by having appropriate training. This study demonstrated significant improvement in knowledge for responding to a diabetic 
emergency and increased perceived self-efficacy following a theory based diabetes management-training program.

As medical and pharmaceutical technology evolve and school budgets are stretched, school districts may fail to adopt appropriate policies and guidelines in a timely manner that address the health needs and care required by the students. School nurses are the most qualified to stay abreast of medical advances and health care trends and to lobby school districts to respond appropriately to the health conditions and health care needs of families and students. 
Reference List

American Academy of Pediatrics. (2001). Guidelines for emergency medical care in school. Pediatrics, 107, 435-436.

American Diabetes Association. (2001). Care of children with diabetes in the school and day care setting. Diabetes Care, 24, 108-112.

American Diabetes Association. (2003). Children with diabetes: A new guide will help schools care for students who have diabetes. Diabetes Forecast, 56, 109-110. Bandura, A. (1994). Self-efficacy. Encyclopedia of Human Behavior, 4, 71-81. New York: Academic Press. Retrieved December 1, 2005 from http://wwwemory.edu/EDUCATION/mfp/BanEncy.html

Bandura. A. (2001). Social cognitive theory: An agentic perspective. Annual Review of Psychology. Retrieved December 1, 2005 from http://www.findarticles.com/cf 0/ m0961/2001_Annual/73232700/print.jhtml

Centers for Disease Control and Prevention. (2002). Facts about diabetes. Retrieved September 1, 2005, from http://www.cdc.gov/diabetes/pubs/estimates.htm Corbett, C. F. (1999). Research-based practice implications for patients with diabetes. Part II: Diabetes self-efficacy. Home Healthcare Nurse, 17, 587-595.

Hayes-Bohn, R., Neumark-Sztainer, D., Mellin, A., \& Patterson, J. (2004). Adolescent and parent assessments of diabetes mellitus management at school. Journal of School Health, 74, 166-169.

Jameson, P. L. (2004). Developing diabetes training programs for school personnel. School Nurse News, 21, 14-17. 
Juvenile Diabetes Research Foundation International. (2005). Laws protecting children with diabetes. Retrieved December 1, 2005 from http://www.jdrf.org/index.cfm?page_id=103473

Lewis, D. W., Powers, P. A., Goodenough, M. F., \& Poth, M. A. (2003). Inadequacy of in school support for diabetic children. Diabetes Technology and Therapeutics, 5, 45-56.

Litarowsky, J. A., Murphy, S. O., \& Canham, D. L. (2004). Evaluation of an anaphylaxis training program for unlicensed assistive personnel. The Journal of School Nursing, 20, 279-284.

National Diabetes Education Program. (2003). Retrieved December 1, 2005 from http://ndep.nih.gov/diabetes.youth/youth_FS.htm

Nichols, P. J., \& Norris, S. L. (2002). A systematic literature review of the effectiveness of diabetes education of school personnel. The Diabetes Educator, 28, 405-414.

Reutzel, T. J., \& Patel, R. (2001) Medication management problems reported by subscribers to a school nurse listserv. Journal of School Nursing, 17, 131-139.

Siminerio, L., \& Koerbel, G. (2000). A diabetes education program for school personnel. Practical Diabetes Institute, 17, 174-177.

Thies, K. M. (1999). Identifying the educational implications of chronic illness in school children. Journal of School Health, 69, 392-397.

VanCura, J., \& Gunchick, D. (1997). Five key components for effectively working with unlicensed assistive personnel. MedSurg Nursing, 6, 270-275.

Zombeck, M. (2001). Pediatric education for diabetes in schools. PADRE Foundation www.pedsonline.org 
Table 1. Comparison of the pre and posttest results for knowledge and self-efficacy

\begin{tabular}{|c|c|c|c|c|}
\hline Instrument & Mean (SD) & $N$ & $d f$ & $t$ \\
\hline Pretest knowledge score & $7.1500(1.84320)$ & 20 & & \\
\hline Posttest knowledge score & $8.0500(1.19097)$ & 20 & & \\
\hline Paired differences between & & & & \\
\hline pre- \& posttest knowledge scores & -.90000 & & 19 & -2.349 \\
\hline Preself-efficacy & $21.8500(7.64526)$ & 20 & & \\
\hline Postself-efficacy & $14.2000(3.77805)$ & 20 & & \\
\hline Paired differences between & & & & \\
\hline Pre- \& postself-efficacy scores & 7.65000 & & 19 & 5.691 \\
\hline
\end{tabular}

Note. Paired $t$-tests were used for comparisons. $P<0.05$, two-tailed. 